\title{
LA EVOLUCIÓN DE LA MASCULINIDAD EN DISNEY A TRAVÉS DE LA BELLA Y LA BESTIA: DE LA VERSIÓN ANIMADA DE KIRK WISE Y GARY TROUSDALE (1991) A LA DE ACCIÓN REAL DE BILL CONDON (2017)
}

(iD) Nerea Cuenca Orellana*

\section{Resumen}

El clásico Disney La Bella y la Bestia (Kirk Wise y Gary Trousdale, 1991) es considerado un icono de la cultura popular, que no sólo fue un éxito de taquilla en el momento de su estreno, sino que se convirtió en el primer largometraje animado en ser nominado al Óscar a la Mejor Película. Casi treinta años después, en 2017, el mismo estudio realizó su remake en acción real dirigido por Bill Condon, alcanzando de nuevo una gran audiencia y diversas nominaciones debido a su calidad estética y de producción. Apoyándose en los estudios de género y utilizando una metodología centrada en el aspecto psicológico del personaje de ficción, en este artículo se analiza la representación de la masculinidad en ambas películas con el objetivo de observar su evolución y, especialmente, qué discursos articulan este tipo de textos audiovisuales que tienen la capacidad de llegar a todos los públicos. Bestia.

Palabras clave: Masculinidad, estudios de género, personaje, Disney, La Bella y la

\section{Resumo}

A evolução da masculinidade na Disney por meio da Bela e o Monstro: da versão animada de Kirk Wise e Gary Trousdale (1991) à versão live action de Bill Condon (2017)

O clássico da Disney A Bela e o Monstro (Kirk Wise e Gary Trousdale, 1991) é considerado um ícone da cultura popular, que não só foi um êxito de bilheteira na época do seu lançamento, mas também se tornou a primeira longa-metragem de animação a ser nomeada para o Óscar de Melhor Filme. Quase trinta anos depois, em 2017, o mesmo estúdio fez o seu remake em live action, realizado por Bill Condon, alcançando novamente um grande público e várias nomeações devido à sua qualidade estética e de produção. Apoiando-se

Universidad Rey Juan Carlos (URJC), Camino del Molino s/n, 28943 Fuenlabrada, Madrid, España.

Dirección postal: Los Arcos 3, CP 28971 - Griñón, Madrid, España.

Correo electrónico: nerea.cuenca@urjc.es

Universidad Carlos III de Madrid (UC3M), 28903 Getafe, Madrid, España.

Dirección postal: Edificio Ortega y Gasset, Calle Madrid, 126, 28903 Getafe, Madrid, España.

Correo electrónico: nmperez@hum.uc3m.es 
nos estudos de género e utilizando uma metodologia focada no perfil psicológico da personagem ficcional, este artigo analisa a representação da masculinidade em ambos os filmes com o objetivo de observar a sua evolução e, principalmente, que discursos articulam este tipo de textos audiovisuais que têm a capacidade de chegar a todos os públicos.

Palavras-chave: Masculinidade, estudos de género, personagem, Disney, A Bela e o Monstro.

\begin{abstract}
The Evolution of Masculinity at Disney through Beauty and the Beast: From the animated version of Kirk Wise and Gary Trousdale (1991) to the live action version of Bill Condon (2017)

Disney's classic Beauty and the Beast (Kirk Wise and Gary Trousdale, 1991) is considered an icon of popular culture, which was not only a box office success at the time of its release, but also became the first animated feature film to be nominated to an Academy Award for Best Picture. Almost thirty years later, in 2017, the same studio made a live-action remake directed by Bill Condon, reaching a large audience and obtaining several nominations due to its aesthetic and production quality. From a gender studies approach and applying a methodology focused on the psychological profile of the fictional character, this article analyzes the representation of masculinity in both films. The aim is to examine its evolution, and especially the discourses conveyed by audiovisual texts that are able to reach all kinds of audiences.
\end{abstract}

Keywords: Masculinity, gender studies, character, Disney, Beauty and the Beast.

\title{
1. Introducción: El arco de transformación en el cine de animación
}

En las historias audiovisuales se representan, a través de los personajes, iconos masculinos y femeninos como ideales a seguir. Una historia comienza cuando un personaje lleva a cabo acciones para conseguir un objetivo cualquiera, pero que para él/ella es difícil de lograr (Sanz Magallón 2007). El personaje es una pieza esencial en todo guion, dado que la historia que cuenta se centra en sus necesidades, sus objetivos internos y sus metas externas. El esfuerzo que realiza y las decisiones que toma dicho personaje - para superar o aliviar estos tres puntos centrales de la narración - es lo que resalta el valor de la historia. En definitiva, lo que se busca es que los personajes tengan personalidad y vivan emociones (Porter 2000). Es importante que estos tengan personalidad para que la espectadora o el espectador vaya descubriendo al personaje y conozca así sus valores, sus miedos, sus virtudes y sus defectos. Estos son los elementos que los "humanizan" y, por tanto, los que invitan a que la audiencia pueda identificarse con ellos.

Seymour Chatman describió el concepto "personaje" como una unidad psicológica y de acción que debe ser estudiada como una categoría narrativa individual, en la que se combinan una serie de rasgos y características diferenciales que hacen único a este sujeto $(1990,155)$. Los personajes pueden ser materia narrativa en tanto se dejan modelar en función de la acción o pueden ser considerados como 
conciencia narrativa porque participan en la forma de narrar (Anderson 2007, 237). En cualquiera de los dos casos, el personaje o agente de la acción solamente existe en relación a la acción: lo que hace y cómo se comporta, lo que dice, con quién y cómo se relaciona. Son los rasgos y características de un personaje en cuestión lo que le hacen único, independientemente de cuál sea la forma en que se presenten: humana, animal, monstruo, criaturas sobrenaturales o formas abstractas (ibíd., 237-238).

Pero estos rasgos y características no son estáticos. A lo largo de la acción, el personaje puede mutar y adquirir comportamientos y formas de expresarse completamente distintas a lo que se apreció al inicio. Esto es lo que se conoce como "arco de transformación" de un personaje. Los arcos de transformación de las historias se pueden categorizar en cinco tipos de transformaciones interiores: el arco plano, la transformación radical, los arcos moderados, los arcos traumáticos y las transformaciones circulares del personaje (Sánchez-Escalonilla 2014). Como señala Linda Seger (1991, 182), la transformación interior o arco de transformación surge cuando el personaje no está seguro de sí mismo porque es entonces cuando vive un conflicto interno.

En el caso de las historias del cine de animación, en los últimos treinta años estas se han centrado más en generar interés por las crisis internas que sufren los personajes que por crear conflictos externos. Los deseos y las necesidades que les van surgiendo, la toma de decisiones y aprender a afrontar cualquier situación frente a la resolución de los conflictos generan crisis internas que les hacen evolucionar (Finch 2011). "El conflicto es el elemento clave de cualquier forma dramática" (Seger 1991, 181). Los conflictos pueden ser de relación, sociales o de situación. Se introdujeron en la animación las crisis personales de los personajes, lo que diferenciaba a los nuevos personajes de aquellos que se crearon cincuenta años atrás (Finch 2011). Esto ha repercutido en el diseño de las personalidades, que pretende alejarlos de los estereotipos literarios clásicos (Martínez González 2011). Los arquetipos siguen existiendo, pero sus personalidades son mucho más variadas de lo que los cuentos de hadas permitían (Martínez González 2011).

\section{La masculinidad en la animación del estudio Disney}

El arco de transformación es clave para presentar un nuevo ideal de masculinidad o de feminidad a la sociedad a través de personajes protagonistas. La masculinidad aparece reforzada por los medios de comunicación, que, para ello, infravaloran lo femenino (Morales Romo 2015, 36-37). En la cultura occidental los varones siguen trabajando con un esquema tradicional narrativo con el que se enseña a las mujeres a aceptar la dominación masculina. Esta tradición aparece en las adaptaciones de Disney (Downey 1996, 189). En el mundo Disney, los padres ejercen el dominio y las madres no aparecen en las historias con protagonistas 
femeninas y, si lo hacen, no es como co-protagonistas ni aportan acción en la narración. Este punto de vista masculino promueve "el patriarcado", en el sentido de que impone una necesidad de la figura paterna como establecimiento del orden: primero en la representación del cuento y, posteriormente, en las adaptaciones Disney (Zipes 2011, 189).

Curiosamente, se considera que los personajes femeninos de Disney ${ }^{1}$ son un modelo para ambos sexos, mientras que es más difícil sentirse representado por un personaje masculino de todos los creados por el estudio, tanto en la Época Clásica (1937-1967) como en la Post-Walt (1968-1988) (Yerby et al. 2014). Este punto de vista cambia cuando se inicia el periodo conocido como Renacimiento de la animación (1989-1999). Si bien el primer personaje masculino de este periodo, el personaje del príncipe Eric de La Sirenita (Ron Clements y John Musker, 1989), es plano y no evoluciona siguiendo la estela de personajes masculinos anteriores de Disney, tales como el príncipe de Blancanieves y los siete enanitos (David Hand, 1937), del príncipe de La Cenicienta (Clyde Geronimi, Wilfred Jackson, y Hamilton Luske, 1950) o del príncipe Felipe en La bella durmiente (Clyde Geronimi, 1959) (Cuenca Orellana 2019, 189).

A partir de 1991, Disney aumenta el protagonismo y, por tanto, la acción de sus personajes masculinos. Desde ese momento, el género de animación deja de ser un método de entretenimiento infantil para dar paso a nuevas temáticas, las cuales impulsan a todos los públicos a mostrarse interesados en ver películas de animación (Sánchez Moreno 2009, 5). Al mismo tiempo, se abrió un camino con nuevas opciones de masculinidad gracias a las novedades introducidas en la sociedad. El acceso de las mujeres a la vida laboral implicó también el cambio de los varones en la esfera privada al demandar estas su implicación en el cuidado de los hijos. Estas transformaciones sociales en la década de los 70 en Estados Unidos se vieron reflejadas en la creación de personajes masculinos del cine (Kreimer 1991, 160-161). Esta nueva masculinidad se reflejó en largometrajes como Kramer contra Kramer (Robert Benton 1979), Rain Man (Barry Levinson 1988) o Memorias de África (Sydney Pollack 1985). En todas ellas, los personajes masculinos viven un viaje del héroe relacionado con la superación de conflictos en las relaciones sociales - amo-

\footnotetext{
En la presente investigación, cuando se indica "películas o largometrajes Disney", se hace referencia a "películas del estudio de animación Walt Disney". Cabe diferenciar entre Walt Disney Company, y por extensión Walt Disney Pictures, de lo que es Walt Disney Animation Studios. La primera, como multinacional, aglutina otras productoras además de Walt Disney Animation Studios, como Pixar Animation Studios, DisneyToon Studios, Marvel Studios, Lucasfilm, 20th Century Studios, Searchlight Pictures y Blue Sky Studios, entre otras franquicias. En este sentido, quedan fuera de esta investigación otros modelos de masculinidad distintos e interesantes, presentes en otros largometrajes del estudio como el mostrado en Brave (Mark Andrews y Brenda Chapman 2012). Para un análisis de estas películas, incluido el de Brave, véase La construcción del género en las películas de Pixar Animation Studios entre 1995 y 2015: modelos de masculinidad, feminidad y relaciones entre personajes (Cuenca Orellana 2019).
} 
rosas, familiares y amistosas - independientes del vínculo laboral, que constituía una novedad.

En la década de los noventa, el estudio Disney, a pesar de seguir adaptando los relatos para el público infantil donde las protagonistas femeninas sufrían distintas desgracias y eran rescatadas por apuestos y heroicos príncipes, abandonó el referente masculino individualista y simplista de los cuentos de hadas. Así, para introducir nuevas características al héroe tradicional, los creativos de Disney se fijaron en las películas de acción real de Hollywood que ya contaban con una masculinidad más social, puesto que tradicionalmente la masculinidad se ha relacionado con el aislamiento y el aparente desinterés por el manejo de sentimientos y emociones (Sharma y Das 2016, 10). Este periodo renacentista se caracteriza por el estreno de diez largometrajes donde los personajes masculinos - Bernardo en Los rescatadores en Cangurolandia (Hendel Butoy y Mike Gabriel 1990), Bestia en La bella y la bestia (Kirk Wise y Gary Trousdale 1991), Aladín en Aladin (Ron Clements y John Musker 1992), Simba en El rey león (Rob Minkoff y Rogers Allers 1994), John Smith en Pocahontas (Mike Gabriel y Eric Goldberg 1995), El jorobado de Notre-Dame (Kirk Wise y Gary Trousdale 1996), Hércules (Ron Clements y John Musker 1997), Capitán Li Shang en Mulán (Tony Bancroft y Barry Cook 1998) y Tarzán en Tarzán (Kevin Lima y Chris Buck 1999) - dejan claro que son los héroes de la acción, incluso cuando ellas son, aparentemente, el centro de la historia. La forja heroica recae en ellos, puesto que o las rescatan o bien influyen de manera positiva en la evolución interna que ellas viven. La desigualdad de género aparece en las narraciones audiovisuales en términos cuantitativos - más personajes masculinos que femeninos -, pero también a la hora de repartir los papeles en cuanto a la superación de conflictos y la resolución final del mismo u objetivo narrativo (VázquezMiraz 2017, 67). En la última década, la seguridad atribuida a los personajes masculinos eclipsa a los femeninos, por lo que se perpetúa una cultura androcéntrica copiada de los cuentos de hadas (Cantillo Valero 2010, 2).

Esta desigualdad continúa presente en los últimos años, desde lo que algunos autores han denominado como "etapa revival de las princesas de Disney" (Cantillo Valero 2018), y que comenzaría en 2009. Así, con el estreno de Tiana y el sapo (John Musker y Ron Clements 2009) comienza un nuevo periodo en el que, aparentemente, las princesas no necesitan ser rescatadas, sino que son heroínas que saben defenderse ante cualquier villano/a para conseguir su objetivo dramático, el cual ya no es casarse con el héroe. Es en este periodo cuando Disney decide lanzar sus grandes éxitos en acción real - esto es, sin dejar atrás la animación, pero buscando nuevos éxitos de taquilla y conquistando al público adulto que disfrutó de las versiones animadas durante su infancia.

Si bien ya había habido adaptaciones en acción real como El libro de la selva: la aventura continúa (Stephen Sommers 1994) y 101 Dálmatas (Stephen Herek 1996), no es hasta 2010 con Alicia en el país de las maravillas, dirigida por Tim Burton, un director-autor de prestigio, cuando Disney inaugura y consolida la tendencia de 
los remakes de sus clásicos con actores de carne y hueso: El aprendiz de brujo (Jon Turteltaub 2010), a partir del cortometraje homónimo incluido en Fantasía (Bill Roberts y otros 1940), al que seguiría Maléfica (Robert Stromberg 2014) y La Cenicienta (Kenneth Branagh 2015). Al mismo tiempo, la evolución de la tecnología CGI (Computer Generated Imagery / imágenes generadas por ordenador) y su utilización en cine ampliaron las posibilidades de crear remakes de live action (acción real) sin límites a la imaginación, como sucedió con El Libro de la Selva (Jon Favreau 2016). También ese mismo año se estrenaría Peter y el dragón (David Lowery 2016) y Alicia a través del espejo (James Bobin 2016). El resto de adaptaciones hasta la actualidad han sido: La bella y la bestia (Bill Condon 2017), Christopher Robin (Marc Forster 2018), Dumbo (Tim Burton 2019), Aladin (Guy Ritchie 2019), El Rey León (Jon Favreau 2019), Maléfica: Maestra del Mal (Joachim Rønning 2019), La Dama y el Vagabundo (Charlie Bean 2019) y Mulán (Niki Caro 2020).

\section{Objetivos y justificación}

Este trabajo tiene como objetivo analizar el ideal de masculinidad que ha articulado el estudio Disney en los últimos treinta años, utilizando como estudio de caso el personaje protagonista Bestia en las películas La bella y la bestia: el largometraje de dibujos animados de 1991 dirigido por Kirk Wise y Gary Trousdale, y la versión de acción real de 2017 de Bill Condon.

La historia, basada en la versión que Jeanne-Marie Leprince de Beaumont escribió en 1770 a partir de la original de Gabrielle-Suzanne Barbot de Villeneuve, cuenta la historia de Bella, una joven brillante que sueña con aventuras más allá de los confines de su pueblo en Francia. Bella no quiere saber nada del engreído Gastón, quien la persigue sin descanso. Todo cambia un día cuando su padre Maurice es encarcelado en el castillo de una horrible Bestia, y Bella se ofrece a intercambiarse con su padre quedando recluida. Rápidamente se hace amiga del antiguo personal del lugar, que fue transformado en objetos tras una maldición. Bella consigue que Bestia se enamore de ella, rompiendo así el hechizo y devolviendo a todos a su forma humana, incluida la Bestia que se convierte en un hermoso príncipe.

Se ha elegido este personaje por varios motivos. En primer lugar, por ser un personaje masculino con gran peso narrativo, característica que, como se ha visto anteriormente, es novedosa en el estudio Disney. En ese sentido, mientras que en el cuento de hadas se trata la historia de una joven, en el largometraje de Disney la historia relata la transformación de la Bestia. En la historia de Beaumont no se sabe de qué trata el hechizo hasta que Bestia captura al padre de Bella (Downey 1996). Así, la película otorga un mayor valor al personaje masculino. Por este motivo, hay autoras que consideran que las historias de Disney de los años 90 dan más protagonismo y heroicidad al personaje masculino de lo que se había hecho anteriormente (Jeffords 1995, 171). 
En segundo lugar, por considerar a la Bestia como un icono de la cultura popular consolidado, pues ambas películas tuvieron un éxito de público y también de taquilla. En el primer caso, fue un éxito paradigmático, recaudando más de 171 millones de dólares en EEUU y más de 403 millones en todo el mundo². Su número de ventas hizo que fuera la tercera película más exitosa de 1991, y el primer largometraje de animación que alcanzó los 100 millones de dólares, formando parte del Top 10 del American Film Institute. Además, obtuvo tres Globos de Oro (Mejor Película Comedia o Musical, Mejor BSO, Mejor Canción Original), y fue galardonada con dos Óscar: a la Mejor Banda Sonora Original y a la Mejor Canción Original, recibiendo un total de seis nominaciones, convirtiéndose así en la primera película animada nominada al Óscar a la Mejor Película, ya que en ese momento aún no existía la categoría de Óscar a la Mejor Película de Animación. En 2002 fue añadida al Registro Nacional de Películas de Estados Unidos como "cultural e históricamente significativa".

En cuanto a la versión de Bill Condon, el tráiler oficial alcanzó 91,8 millones de visitas, lo que superó el número de puntos de visitas vistas en esa cantidad de tiempo en la historia, incluyendo para los resúmenes de otras películas distribuidas por Disney como Star Wars: Episodio VII. El despertar de la Fuerza (J.J. Abrams 2015). Alcanzó 1.260.998.471 dólares a nivel mundial, convirtiéndose en la décima tercera película más exitosa de todos los tiempos y la segunda de $2017^{3}$. Cabe destacar que fue la película de imagen real de Disney más exitosa, sólo superada por el estreno del remake en imagen real de El Rey León de 2019, que la superó en taquilla. Fue nominada a los Premios Óscar en las categorías de Mejor Diseño de Producción y Mejor Vestuario.

Este trabajo pretende determinar cómo es la masculinidad que el estudio Disney representa y si ha variado en las últimas tres décadas. Con este análisis se busca, además, concretar qué cualidades masculinas siguen presentes y cómo la evolución interior que sufre el personaje objeto de estudio está completamente relacionada con la búsqueda de una masculinidad amable, emocional y sentimental como características que las mujeres buscan en los varones y, por tanto, como rasgos fundamentales para que la conquista del personaje femenino tenga lugar en la narración. Por tanto, se busca comprobar si ese ideal de masculinidad se ha visto alterado en el arco temporal que va de un estreno a otro. Para tal fin, la investigación se centra en el arco de transformación y las relaciones del personaje. Tras el análisis se realizará una comparación con la que determinar si ha evolucionado la imagen masculina que Disney nos presenta en los últimos años respecto a la que representaba en la última década del siglo XX.

Véase: https:/ / www.boxofficemojo.com/release/rl4490753/weekend/ (Acceso: 20/12/2020). Véase: https: / / www.boxofficemojo.com/release/rl222594561/ (Acceso: 20/12/2020). 


\section{Metodología}

Se ha optado por una metodología centrada en el aspecto psicológico del personaje. En ese sentido, con el tiempo, los héroes masculinos Disney han ido ganando una mayor participación narrativa, no sólo en cuanto a tiempo de aparición, sino también al aumentar su profundidad psicológica en el transcurso de la acción narrativa (Do Rozario 2004). Lo que, en películas de la Época Clásica, Post-Walt o La Sirenita en el Renacimiento, era un amor a primera vista basado en el físico y la idealización del otro, derivó en un viaje interior de los dos enamorados a partir de 1991. La primera película que contó con este cambio fue La bella y la bestia. Mediante el arco de transformación de ambos personajes, se profundizó en los dos puntos de vista, y, por tanto, en la psicología y en la manera de aceptar los pensamientos, opiniones y acciones de su pareja. Los cambios en la manera de iniciar su relación determinan una nueva psicología de los personajes, que tiene un mayor sentido en la época de creación. Esto, precisamente, facilitó una mayor identificación con los personajes por parte del espectador (Rodríguez y Cardete 2018, 98).

Para profundizar en la psicología del personaje objeto de estudio y de su remake, se ha utilizado la ficha de análisis de Carlos Grossocordón (2019). Con las variables recogidas en esta tabla se pretende definir cómo ha evolucionado psicológicamente el personaje desde 1991 hasta 2017 y comprobar si han variado o no las características que componen la masculinidad del estereotipo new man o si ha surgido un nuevo icono representativo masculino.

\section{Ficha de análisis de la dimensión psicológica de un personaje}

\begin{tabular}{l}
\hline Arco de transformación \\
\hline Ambiciones \\
\hline Objetivos primarios/secundarios \\
\hline Tipo de conflicto \\
\hline Frustraciones \\
\hline Temperamento/actitud ante la vida \\
\hline Carácter/tendencias \\
\hline Virtudes \\
\hline Defectos \\
\hline Carisma \\
\hline Vida afectiva \\
\hline Autoestima/complejos \\
\hline Supersticiones \\
\hline Imaginación \\
\hline Inteligencia \\
\hline Fuente: Grossocordón (2019)
\end{tabular}




\section{Resultados y discusión}

\section{Ficha 1:}

Análisis de la dimensión psicológica de Bestia 1991

\begin{tabular}{|c|c|}
\hline Arco de transformación & Transformación radical \\
\hline Ambiciones & Ninguna \\
\hline Objetivos primarios/secundarios & Dejar de tener aspecto físico de Bestia \\
\hline Tipo de conflicto & Interno \\
\hline Frustraciones & $\begin{array}{l}\text { No poder salir de ese cuerpo en el que se siente } \\
\text { encerrado }\end{array}$ \\
\hline Temperamento/actitud ante la vida & Agresivo, violento, solitario \\
\hline Carácter/tendencias & Malhumorado \\
\hline Virtudes & Gentil y dulce cuando Bella muestra interés en conocerlo \\
\hline Defectos & Testarudo \\
\hline Carisma & $\begin{array}{l}\text { De autoridad, excepto cuando se enamora de Bella, } \\
\text { con quien muestra su carisma de bondad }\end{array}$ \\
\hline Vida afectiva & Se enamora de Bella \\
\hline Autoestima/complejos & $\begin{array}{l}\text { Tiene poca autoestima y está lleno de complejos por } \\
\text { su aspecto físico }\end{array}$ \\
\hline Supersticiones & No se aprecian \\
\hline Imaginación & No se aprecia \\
\hline Inteligencia & $\begin{array}{l}\text { Ha desarrollado su inteligencia física: sabe pelear } \\
\text { contra los lobos y contra Gastón. No tiene inteligencia } \\
\text { emocional porque no sabe cómo tratar a Bella hasta } \\
\text { que los habitantes del castillo le aconsejan que sea } \\
\text { dulce y delicado }\end{array}$ \\
\hline
\end{tabular}

\section{Ficha 2:}

Análisis de la dimensión psicológica de Bestia 2017

\begin{tabular}{ll}
\hline Arco de transformación & Transformación radical \\
\hline Ambiciones & $\begin{array}{l}\text { En la primera secuencia se descubre que le encan- } \\
\text { ta hacer fiestas con mucha gente en su casa, según } \\
\text { avanza la trama, se descubre que conquistar a Bella se } \\
\text { convierte en una ambición }\end{array}$ \\
\hline Objetivos primarios/secundarios & $\begin{array}{l}\text { Abandonar su forma de bestia para volver a ser un } \\
\text { príncipe, para ello necesita que una chica se enamore } \\
\text { de él }\end{array}$ \\
\hline & \multicolumn{1}{c}{ (continua na página seguinte) }
\end{tabular}




\begin{tabular}{ll}
\hline Tipo de conflicto & Interno \\
\hline Frustraciones & $\begin{array}{l}\text { Su madre murió siendo él niño y ese dolor le hace } \\
\text { poner barreras con el resto del mundo, ser déspota y } \\
\text { arrogante y no sentir amor hasta conocer a Bella }\end{array}$ \\
\hline Temperamento/actitud ante la vida & Violento, egoísta y prepotente \\
\hline Carácter/tendencias & $\begin{array}{l}\text { Se enfada con facilidad, maltrata, grita e incluso, hu- } \\
\text { milla, es solitario y superficial hasta que Bella pone } \\
\text { interés en él y comienza a mostrarse amable, empático } \\
\text { y considerado }\end{array}$ \\
\hline Virtudes & $\begin{array}{l}\text { Culto, sensible y cercano con Bella cuando la va } \\
\text { conociendo (deja salir su yo oculto según avanza la } \\
\text { narración) }\end{array}$ \\
\hline Defectos & Temperamento fuerte y quiere salirse con la suya \\
\hline Carisma & $\begin{array}{l}\text { De autoridad, excepto cuando se enamora de Bella, } \\
\text { con quien muestra su carisma de bondad }\end{array}$ \\
\hline Vida afectiva & Se enamora de Bella y es correspondido \\
\hline Autoestima/complejos & $\begin{array}{l}\text { Tiene la autoestima baja y está lleno de complejos por } \\
\text { la maldición que sufre, pero poco a poco los va supe- } \\
\text { rando con Bella a su lado }\end{array}$ \\
\hline Supersticiones & No aparecen \\
\hline Imaginación & $\begin{array}{l}\text { No parece imaginativo antes de conocer a Bella ni } \\
\text { cuando la concibe como el objeto de deseo con el que } \\
\text { cambiar su vida, sin embargo, cuando se interesa por } \\
\text { ella como persona, usa su imaginación para buscar } \\
\text { actividades que sean del interés de la muchacha: leer, } \\
\text { pasear por el jardín, viajar al pasado... }\end{array}$ \\
\hline $\begin{array}{l}\text { Ha desarrollado su inteligencia física: sabe pelear con- } \\
\text { tra los lobos y contra Gastón. No es nada inteligente } \\
\text { en términos emocionales, puesto que no ha sabido } \\
\text { gestionar el dolor de la pérdida de su madre }\end{array}$ \\
\hline
\end{tabular}

Observando los resultados de este análisis comparativo, se puede indicar en primer lugar que en la personalidad de Bestia de 1991 se incluyó un aspecto clave para acercarlo al mundo real: es capaz de mostrar emociones y sentimientos complejos que le llevan tener mal carácter cuando se enfada, pero, también, a mostrar su buen humor e, incluso, su lado inocente (Fonte 2001). Sin duda, se creó un personaje en el que el buen trato hacia Bella aparece paulatinamente, según va descubriendo a la muchacha como persona y ella se interesa por él. Este conocimiento mutuo a él le influye positivamente, porque comienza a demostrar su respeto por las decisiones y la personalidad de la joven. Así, Bestia se aleja del icono masculino tradicional representado por Gastón, el villano de la película, quien pretende imponer el matrimonio como única solución. Y es que fue a partir de 1991 cuando 
Disney añade nuevas características al héroe o personaje protagonista, el cual se estereotipa con lo que se definió como "hombre nuevo" (new man).

Este nuevo ideal de masculinidad que aparece en los dibujos animados se centra en aprender a ser humano, cercano, social y capaz de desarrollar con éxito las relaciones familiares, de amistad y de pareja, sin abandonar el rol de salvador y protector. Con estas cualidades, Disney crea personajes masculinos que siguen el nuevo estereotipo masculino: el new man (Guarinos 2009, 282). Una de las características que componen este nuevo ideal es que Bestia aprende a amar y demostrar sus sentimientos en público. Este nuevo icono masculino se interesa por las emociones, las decisiones y los pensamientos de quienes le rodean, en especial, de la chica de quien se enamora. Este estereotipo masculino también puede denominarse "nuevos liberales" (Martín 2002, 13), que son varones que desean ser compañeros y padres modelo que, a través de las relaciones amorosas y familiares, alcanzan su propia plenitud y madurez.

Bestia del 1991 experimenta un gran aprendizaje a lo largo de la película, quien pasa de ser egoísta e individualista a un new man gentil y considerado cuando deja de interesarse por Bella como el objeto de deseo con el que romper el hechizo que le devolverá su físico de apuesto príncipe. Con el maleficio, el físico de Bestia (cabeza de león, cuerpo de oso, patas de lobo, cuernos de búfalo...) refleja cómo es el caos de su interior, pero cuando comienza a mostrarse tal y como es con Bella, su transformación interior y el beso de ella acaban dando lugar a un príncipe alto, rubio y con ojos claros. La evolución interna de Bestia se basa en comprender que su cambio físico no surgirá hasta no ser capaz de mutar su interior. El objetivo de la hechicera es que el protagonista deje atrás la soberbia y las faltas de respeto hacia los demás. En el transcurso de su evolución, Bestia aprende a aceptarse como persona, valorar a los demás y descubrir que no hay que juzgar a nadie por el exterior. En resumen, Bestia es el primer personaje Disney que pasa de ser musculoso, fuerte y dominante como los héroes de acción del Hollywood de los 80, a ser considerado, cariñoso y capaz de autosacrificarse. Como señala Susan Jeffords, "Bestia es un 'hombre nuevo', el único que puede transformarse a sí mismo, pasando de ser fuerte, musculoso y autoritario, característico de los 80, a considerarse un hombre amoroso y capaz de autosacrificarse, propio de los 90" (Jeffords 1995, 170; traducción propia) ${ }^{4}$.

No se ha optado, por tanto, por cambiar el icono representativo masculino con la imagen masculina surgida durante el postfeminismo, ya que, desde 2009, los héroes Disney son representados como cómicos, quizá incluso inmaduros o incapaces de hacer algo por sí mismos, para poder, así, subrayar la idea de capacidad e independencia de los personajes femeninos que han aparecido desde Tiana

"The Beast is The New Man, the one who can transform himself from the hardened, musclebound, domineering man of the ' 80 s into the considerate, loving, and self-sacrificing man of the '90s”' (1995, 170). 
y el sapo. Tampoco se ha representado el nuevo príncipe azul bajo el ideal softman que Disney introdujo en Frozen en 2013. Este softman u "hombre blando" surge, según Elisabeth Badinter, "para gustar a las mujeres que durante los años 70 pusieron en cuestión al macho, algunos hombres creyeron que les convenía abandonar todo tipo de virilidad y adoptar valores y comportamientos tradicionalmente femeninos" (1993, 175).

Ambas películas comienzan con la misma premisa: "Al fin y al cabo, ¿quién querría amar a una Bestia?" Pero el hechizo tiene solución y sabemos desde el principio que, si consigue conquistar a una mujer, se convertirá en príncipe de nuevo, es decir, alcanzará su objetivo dramático. Sin duda, el objetivo dramático de Bestia se cumple progresivamente: poco a poco y gracias a la ayuda de Din Don y de Lumière y a las recomendaciones de la Señora Potts para tratar a Bella, Bestia la conquista con pequeños detalles (pasa tiempo con ella para descubrir sus intereses) y grandes demostraciones (salva a la joven de morir atacada por unos lobos y le permite salir del castillo para cuidar de su padre). Gracias a estas actitudes, la joven se fija en él y descubre que debajo de ese cuerpo hay un corazón noble que necesita amor. Cuando Bella cree que ha perdido a Bestia tras la pelea con Gastón, que le dispara, llora desconsolada.

En La bella y la bestia del 1991 el arco de transformación de Bestia se basa en una transición: de hombre con corazón de bestia a bestia con corazón de hombre. Esto comienza con el hechizo de una bruja que le condena por su incapacidad de amar. Surge entonces el interés del espectador que desea descubrir cómo se resolverá (Jeffords 1995, 167). Mientras que en el cuento de hadas se trata la historia de una joven, en el largometraje de Disney la historia relata la transformación de la Bestia. En la historia de Beaumont no se sabe que es un hechizo hasta que él no captura al padre de Bella. Aun con todo, que Bestia alcance su evolución interior y que la historia se centra, mayoritariamente, en el arco de transformación de él no resta la importancia de Bella en dicha evolución (Cordwell 2016, 10). De hecho, ella es el detonante y se involucra narrativamente en la resolución de los conflictos internos de Bestia (Downey 1996, 91). Se evidencia la agresividad y falta de control de Bestia en escenas en las que la muchacha no respeta las normas que él impone o cuando se muestra testaruda. De este modo, Bestia pierde sus cabales y se torna agresivo. Lo mismo le ocurre cuando Bella acude a la habitación en la que está la rosa encantada, lugar al que Bestia prohibió el acceso. Este comportamiento también se repite cuando la chica decide no bajar a cenar con él. Estos problemas de Bestia se convierten en el centro de la historia (Jeffords 1995, 164).

Es gracias al arco de transformación que vive Bestia a través del enamoramiento de la princesa que se incluye tridimensionalidad psicológica al personaje masculino, y este lo transmite a Bella y la historia de amor que surge entre ambos durante el transcurso de la película (Finch 2011, 293). Y es así también cómo los héroes Disney de los 90 se convirtieron en hombres con más opciones y poder, en una época en la que los cambios culturales, la lucha de las mujeres, los estímulos 
políticos-sociales y la voluntad individual y grupal generaron modificaciones hacia el trato igualitario (Bonino 1999, 20).

Bestia abandona de forma paulatina comportamientos de la masculinidad tradicional: la autonomía, la autoridad, la agresividad o el egoísmo. Actitudes por las que fue castigado con el hechizo, pero que no supera hasta conocer a Bella. A través de la convivencia, se crea un vínculo entre los dos. Una vez que Bestia se acepta a sí mismo, deja paso a las relaciones sociales y amorosas, al diálogo y el aprendizaje de preocuparse por los demás y ofrecerles ayuda (aprende a dar de comer a los pájaros bajo la nieve). Así, a través de las relaciones, Bestia descubre qué es la generosidad y la pone en práctica (le muestra a Bella toda la biblioteca para que ella pueda leer) y comprender la importancia y necesidad de libertad de Bella para decidir si quedarse a su lado o no. Por último, Bestia aprende a valorar a Bella por cómo es, en lugar de exigirle un beso que le libere, como ocurría en historias previas como La bella durmiente o Blancanieves y los siete enanitos.

Bestia de 2017 está basado en la versión que Disney hizo en 1991; sin embargo, la inclusión de dos nuevas secuencias interpretadas por el personaje de Bestia dan una visión más amplia de quién es y qué supone su cambio. Primero hacia un "príncipe déspota y consentido", capaz de cargar a su pueblo de impuestos para comprar los objetos más caros para su castillo, después a héroe actual. Estas novedades añaden profundidad a la psicología del personaje y a su transformación interior: la primera de ellas, tras la muerte de su madre - la persona a la que más amaba - para luego salir de su arrogancia y superficialidad al sentir amor, también por una mujer, pero en este caso, amor romántico. La importancia del amor es, de nuevo, la relación que guía el cambio interior de Bestia. El hecho de que sea el "amor romántico" lo que mueve a Bestia a evolucionar supuso en 1991 toda una modificación narrativa: hasta entonces, quien cambiaba de vida por amor era el personaje femenino, salvado de las garras de la bruja por el héroe. Es así como se aprecia que la relación amorosa que se desarrolla entre Bella y Bestia genera identidad, supone compañía y aporta seguridad emocional al personaje masculino en ambas películas. Esta relación se convierte en una forma de expresión y conocimiento de sí mismo. De hecho, la relación amorosa de Bestia 2017 se representa como una oportunidad para que los protagonistas se muestren y acepten tal y como son (Langford 1999, 3).

En la versión de 1991 la familia como institución no es parte del desarrollo personal de Bestia; sin embargo, en la película de 2017 sí tiene cabida. La estupenda relación que Bestia tiene con su madre le marca positivamente; es su pérdida y la educación que su padre - "tan retorcido como él" - le da lo que le lleva a convertirse en una Bestia por dentro, y esto se acaba reflejando en su físico tras el hechizo.

A pesar de ser huraño, Bestia cuenta con dos buenos consejeros: Din Don y Lumière. Los dos son empleados del castillo, y saben que, aunque no tiene mal fondo, su comportamiento es indebido. Ambos le muestran fidelidad y compren- 
sión, de forma que son lo más cercano a un amigo que Bestia tiene. De esta relación resalta la conversación sobre emociones y sentimientos hacia Bella que mantienen los tres hombres. Una conversación que ya existía en la versión de 1991, pero que en la versión de 2017 adentra al espectador en la necesidad del joven de expresar sus impresiones, inseguridad, dudas y miedos en torno a la correspondencia de Bella, pero también a hacerle daño y que ella se niegue a conocerlo. En ese sentido, la relación de Bestia con otros pares masculinos es interesante, pues esa relación de camaradería es importante en tanto responde al "deseo social de afirmar la identidad masculina" (Badinter 1993, 116).

Bestia es un príncipe del que sólo se sabe que, antes del hechizo, gustaba de celebrar fiestas y bailes, así como tenía cierta afición por la lectura. Esto contrasta con el villano de la película: Gastón. Este ha ido a la guerra, le gusta ir a la tasca y cazar, aborrece la lectura y está seguro de que se casará con Bella sin considerar su opinión. Estos rasgos están asociados a la masculinidad tradicional, pues como se dice en su canción, "no hay otro más macho en el pueblo". Desde este punto de vista, las dos películas siguen los aportes que los estudios sobre el icono masculino de $\operatorname{los} 70$ y 80 :

“la recuperación de modelos alternativos y emergentes masculinos, de nuevas masculinidades, que pusieran en entredicho a los modelos tradicionales de ser hombre, criticando esos modelos que ocasionaron una violencia estructural hacia las mujeres, así como el desarrollo de otro tipo de violencias, incidiendo también en los factores de riesgo que tenía para los propios hombres la adopción de estos modelos" (Pinilla 2012, 22).

El hecho de que sea el villano quien represente estas características refuerza la idea de que la imagen masculina hegemónica es aquella que rechaza lo que Gastón representa con el objetivo de alimentar un icono masculino amable, sensible e interesado por las relaciones amorosas igualitarias. Connell (1987) denomina "masculinidad hegemónica" al modelo predominante, aquella masculinidad que es valorada social y culturalmente como única opción válida y que, a su vez, subordina y desprecia al resto de masculinidades. A pesar de que Bestia parece vivir en primera persona una transformación radical, en la versión de 2017 se muestra cómo en el pasado, al pasar de "príncipe educado y sensible" a "príncipe con corazón de Bestia" (cuando su físico se convierte en el de una Bestia), su corazón muestra su verdadera naturaleza, confirmando que el arco de transformación que sufre a través de su relación amorosa es moderado. 


\section{Conclusiones}

Aunque veintiséis años separen los dos largometrajes de La bella y la bestia analizados, poco ha variado el icono masculino representado. Si bien las representaciones cinematográficas de la masculinidad en el cine de acción real se transformaron de manera profunda a partir de la década de los setenta, el cine de animación no lo puso en práctica hasta los noventa. Aunque la adaptación de 2017 podría haber insertado cambios para mostrar una masculinidad más actualizada, el remake ha seguido fielmente la masculinidad de entonces. Aquella que se corresponde con la figura del new man: un hombre sensible, delicado y lleno de bondad que construye relaciones positivas y se interesa por conquistar a la mujer de sus sueños, que nada tiene que ver ya con la sumisión y la aceptación de la jerarquía que la situaba en un lugar inferior. En ese sentido, huye de una masculinidad hegemónica tradicional, que le permite desarrollar relaciones amorosas igualitarias. Esta investigación subraya cómo en el audiovisual contemporáneo en las representaciones de la masculinidad articuladas por Disney no se han insertado cambios tan profundos como los incorporados en 1991, aunque sí se ha matizado al personaje principal a través del arco de transformación y sus relaciones sociales en la persecución de su objetivo narrativo. En la versión de 2017 se aprecia que continúa la representación del new man de la película de 1991, pero se ha ampliado el desarrollo moral del personaje masculino, dotándole de mayor protagonismo y profundidad psicológica.

\section{Referencias bibliográficas}

Anderson, Enrique. 2007. Teoría y técnica del cuento. Barcelona: Ariel.

Badinter, Elisabeth. 1993. XY La identidad masculina. Madrid: Alianza.

Bonino Méndez, Luis. 1999. "Los varones ante el cambio de las mujeres." Lectora: revista de dones $i$ textualitat, 4: 7-22.

Cantillo Valero, Carmen. 2010. “Alfabetización Audiovisual Crítica. Análisis de los estereotipos sexistas en la filmografía de Disney: de la ingenua Blancanieves a la postmoderna Tiana (1937-2009)." Tesis doctoral. Madrid: UNED.

Cantillo Valero, Carmen. 2018. "Las princesas Disney y la construcción de Humanidades Digitales 'silenciadas' en el cine de animación." Index comunicación, 8 (2): 83-102.

Chatman, Seymour. 1990. Historia y discurso. La estructura narrativa en la novela y el cine. Madrid: Taurus.

Connell, Raewin W. 1987. Gender and Power: Society, the Person, and Sexual Politics. Stanford: Stanford University Press.

Cordwell, Caila Leigh. 2016. “The Shattered Slipper Project: The Impact of the Disney Princess Franchise on Girls Ages 6-12." Selected Honors Theses 57. Lakeland: Southeastern University.

Cuenca Orellana, Nerea. 2019. “La construcción del género en las películas de Pixar Animation Studios entre 1995 y 2015: modelos de masculinidad, feminidad y relaciones entre personajes." Tesis doctoral. Burgos: Universidad de Burgos. 
Do Rozario, Rebecca Anne C. (2004). "The Princess and the Magic Kingdom: Beyond Nostalgia, the Function of the Disney Princess." Women's Studies in Communication, 27 (1). DOI: https: / / doi.org/10.1080/07491409.2004.10162465

Downey, Sharon. 1996. "Feminine Empowerment in Disney's Beauty and the Beast". Women's Studies in Communication, 19 (2). DOI: https: / / doi.org/10.1080/07491409.1996.11089812

Finch, Christopher. 2011. El arte de Walt Disney: De Mickey Mouse a Toy Story. Barcelona: Lunwerg.

Fonte, Jordi. 2001. Walt Disney. El universo animado de los largometrajes 1970-2001. Madrid: T \& B Editores.

Grossocordón, Carlos. 2019. "Propuesta metodológica sobre análisis de personajes en el relato cinematográfico." Communication \& Methods, 1(1): 9-28. DOI: https: / / doi.org/10.35951/ v1i1.18

Guarinos, Virginia. 2009. "Belleza, fealdad y deformidad en producciones Disney." De Blancanieves a High School Musical. I Congreso Internacional de Estética Cinematográfica. Bilbao: UPV/EHU.

Jeffords, Susan. 1995. “The Curse of Masculinity. Disney's Beauty and the Beast." En From Mouse to Mermaid. The Politics of Film, Gender, and Culture, editado por Elizabeth Bell, Lynda Haas y Laura Sells, 161-172. Bloomington: Indiana University Press.

Kreimer, Juan Carlos. 1991. El varón sagrado. El surgimiento de una nueva masculinidad. Buenos Aires: Planeta.

Langford, Wendy. 1999. Revolutions of the Heart. Gender, Power, and the Delusions of Love. Londres: Routledge.

Martín, Sara. 2002. Monstruos al final del milenio. Madrid: Imágica.

Martínez González, Estefanía. 2011. “Los mundos [teóricos] de Coraline: Psicoanálisis, Postfeminismo y Postmodernismo en el cine de animación." Con A de Animación, 1: 79-96. DOI: https: / / doi.org/10.4995/ caa.2011.862

Morales Romo, Beatriz. 2015. "Roles y estereotipos de género en el cine romántico de la última década. Perspectivas educativas." Tesis Doctoral. Universidad de Salamanca.

Pinilla, David. 2012. "Masculinidades: un acercamiento a los grupos de hombres por la igualdad en el estado español." Tesis de Máster. Zaragoza: Universidad de Zaragoza.

Porter, Tom, y Galyn Susman. 2000. "Creating Lifelike Characters in Pixar Movies." Communications of the ACM, 43 (1): 25-29. DOI:10.1145/323830.323839

Rodríguez, José, y María Jesús Cardete. 2018. “La bella y la bestia. Todos somos contadores de cuentos." En Los cuentos infantiles y la transmisión de estereotipos: las creencias limitadoras, editado por María Luisa Fanjul. Madrid: AMEC.

Sánchez-Escalonilla, Antonio. 2014. Estrategias de guion cinematográfico. Barcelona: Ariel.

Sánchez Moreno, Ana Belén. 2009. “El cine/cuento animado o la ruptura del modelo clásico." Área Abierta, 24, 1-10.

Sanz Magallón, Ana. 2007. Cuéntalo bien. El sentido común aplicado a las historias. Madrid: Plot Ediciones.

Seger, Linda. 1991. Cómo convertir un buen guion en un guion inolvidable. Madrid: Rialp.

Sharma, Alankaar, y Arpita Das. 2016. “Men, Masculinities and Violence.” Graduate Journal of Social Science, 12(3): 7-11.

Vázquez-Miraz, Pedro. 2017. "Sexismo en Digimon: quince años de inmovilismo." Femeris 2(1): 67-79. DOI: https: / / doi.org/10.20318/femeris.2017.3549

Yerby, Amanda, Samantha Baron y Youjin Lee. 2014. "Gender Roles in Disney Animation." Assignment for History of Animation course. Washington, D.C.: American University.

Zipes, Jack. 2011. The Enchanted Screen. The Unknown History of Fairy-tale Films. Nueva York: Routledge. 
Nerea Cuenca Orellana. Doctora en Ciencias de la Comunicación y Humanidades por la Universidad de Burgos, y miembro del Grupo de Investigación Visual. Ha publicado artículos en revistas de investigación como Index Comunicación, Comunicación y Género o RAEIC. Desde 2009 hasta 2020 trabajó como apoyo docente en la Universidad Rey Juan Carlos (URJC), y en la actualidad es Profesora Asociada en el departamento de Comunicación y Sociología de la URJC.

Correo electrónico: nerea.cuenca@urjc.es

Natalia Martínez Pérez. Profesora ayudante doctora en el departamento de Comunicación en la Universidad Carlos III de Madrid y miembro del grupo de investigación Televisión-Cine: memoria, representación e industria (TECMERIN). Sus publicaciones más recientes se encuentran en la Revista Panamericana de Comunicación y en el volumen La maternidad en la ficción contemporánea (Peter Lang, 2020).

Correo electrónico: nmperez@hum.uc3m.es

Artículo recibido el 04 de Enero y aceptado para su publicación el 22 de Marzo de 2021. 
